\title{
Evaluating and Modeling Proactive Routing Protocols with Route Stabilities using 802.11 DCF and 802.11e EDCF
}

\author{
M. Umar Khan ${ }^{1}$, Mahmood ul-Hassan ${ }^{2,}$ Abdelrahman Osman ${ }^{3}$, Imad Siraj ${ }^{4}$ \\ ${ }^{14}$ (Center for Advanced Studies in Telecommunications (CAST), COMSATS Institute of Information Technology, \\ 44000, Islamabad, \\ ${ }^{23}$ (Department of Computer Science, Arts and Sciences college in Tabrjal/Al Jouf University, KSA,
}

\begin{abstract}
This paper contributes modeling links and route stabilities in three diverse wireless routing protocols. For this purpose, we select three extensively utilized proactive protocols; Destination-Sequenced Distance Vector (DSDV), Optimized Link State Routing (OLSR) and Fish-eye State Routing (FSR). We also enhance the performance of these protocols by modifying their default parameters. All experiments for evaluating and comparing the performance of protocols are performed in NS-2. Optimization of these routing protocols is done via performance metrics; average Throughput, End to End Delay (E2ED) and Normalized Routing Load (NRL) achieved by them. Default routing protocols DSDV, OLSR and FSR and compared and evaluated with modified versions named as M-DSDV, M-OLSR and M-FSR. Numerical Computations for Route Stabilities of these routing protocols through a mathematical modeled equation is determined and then compared with the results obtained through Simulations in NS-2. Moreover, all-inclusive evaluation and scrutiny of these proactive routing protocols are done under the Mac layer standards 802.11 DCF and 802.11e $E D C F$. In this way both Network and Mac layer exploration has been done under the performance metrics which gives overall performance and tradeoff with respect to full utilization of the available resources of Ad-hoc network high scalability scenario. Routing latency effects with respect to route stabilities and Mac layer standards 802.11 and 802.11e are compared and scrutinized with the tradeoff observed in throughput and in overhead (NRL) generated.
\end{abstract}

Keywords - Diverse wireless, routing protocol, proactive protocols, enhance performance, route statbilities, NS-2, DSDV, OLSR, FSR

\section{INTRODUCTION}

IEEE standard 802.11 at present is significant and trendy access methodology used in wireless communication. It enables speedy and straightforward design of network communication as a de facto MAC standard for LANs, WANs in Small Office Home Office (SOHO) or open-places and to the highest degree facilitated wireless access to the internet. Growing esteem of 802.11 standard, numerous services also improved and requirement for Quality of Service (QoS) become obvious. Hence, therapy to QoS setback in 802.11, enhanced version i.e. 802.11e was anticipated [1]. IEEE standard 802.11e i.e. EDCF (Enhanced Distributed Channel Function) defines numerous QOS parameters to IEEE standard 802.11 Distributed Coordination Function (DCF).

Conferring to $802.11 \mathrm{DCF}$, every station with a fresh data packet equipped to access transmission observers channel activity while waiting for an unused period comparable to a Distributed Inter- Frame Space (DIFS) is sensed and at that point the station transmits. Else, when channel is intuited busy, station initializes its back off timer and delays the transmission access for arbitrarily chosen back off interval so to lessen aggregate of collisions.

The basic access mechanism is illustrated in the Fig.1. The EDCF, 802.11e standard announces service differentiation compared to the DCF standard 802.11 DCF by launching four access categories or classes (ACs) for data priorities [2,3]. Presuming QoS Stations (QSTAs) work under saturated or congested traffic, i.e. each QSTA has a data unit called as MAC Protocol Data Unit (MPDU) to correspond later while closing all succeeded transmissions [4]. Standard 802.11e methodology is formulated on four Access Categories (ACs); Voice, Video, Best Effort, and Background.

\section{ProACTIVE Protocols in Ad-Hoc NeTwork}

DSDV, FSR and OLSR which are proactive in nature are table driven protocols. They update their routing table periodically without demand, so issue of extra band width utilization is occurred. Several methods have designed to compensate this problem. In the Low Scalability region all three protocols performs well, but when we take a look for Medium Scalability region OLSR has better performance. This makes this standard efficient by checking the medium state more rapidly than 802.11 . In this way time consumed for accessing the medium is decreased and more enhanced Throughput is achieved in shorter time than in 802.11 DCF. The 
enhanced version 802.11e EDCF provides small Contention Window (CW) size which helps to access the medium more immediately than in $802.11 \mathrm{e}$ EDCF.

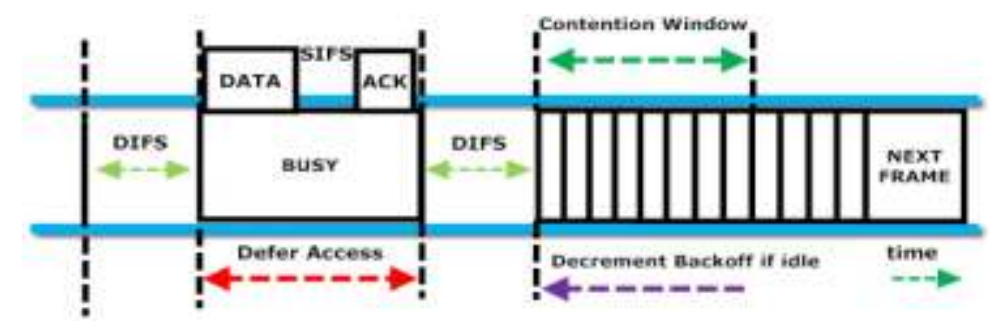

Figure 1: Basic access mechanism 802.11

\subsection{Proactive Protocols in brief}

We have considered three extensively utilized protocols in Ad-hoc Networks; Destination Sequenced Distance Vector (DSDV) [5], Fish-eye State Routing (FSR) [6][7] and Optimized Link State Routing (OLSR) [8][9], are proactive in nature. Altogether, these three proactive protocols practice hop-by-hop routing scheme designed for packet forwarding. In DSDV, packets are disseminated and then path is calculated by Distributed Bellman Ford (DBF) algorithm. In FSR, DBF algorithm is utilized for path calculation. The nodes keep up a table carrying link state information constructed on fresh statistics acknowledged via adjacent nodes. In addition, nodes occasionally interchange it with confined neighbors. Path calculation mechanism in OLSR is carried out through Dijikstra's algorithm. Proactive routing protocols in brief with their features are given in table 1.

Table 1: Proactive routing protocols with their features

\begin{tabular}{|l|c|c|c|}
\hline \multicolumn{1}{|c|}{ Features } & DSDV & OLSR & FSR \\
\hline Path Calculation & DBF Algorithm & Dijikstra's Algorithm & DBF Algorithm \\
\hline $\begin{array}{l}\text { Flooding Control } \\
\text { Mechanism }\end{array}$ & $\begin{array}{c}\text { Exchange topology info } \\
\text { with neighbors }\end{array}$ & $\begin{array}{c}\text { Broadcast via selected } \\
\text { MPRs }\end{array}$ & $\begin{array}{c}\text { Graded Frequency } \\
\text { mechanism }\end{array}$ \\
\hline Overhead Reduction & Incremental updates & MPRs & Fish-eye technique \\
\hline Packet Forwarding & Hop by Hop routing & Hop by Hop routing & Hop by Hop routing \\
\hline Special Features & Route Settling Time & MPRs & Multi-Scope Routing \\
\hline
\end{tabular}

III. RELATED WORK AND SHORTCOMINGS

After the extensive research concerned to our work we have summarized the previously done work and their shortcomings in table 2 , and table 3 shows our proposed work.

Table 2: Related works and its shortcomings

\begin{tabular}{|l|l|l|l|l|}
\hline \multicolumn{1}{|c|}{ Related work } & $\begin{array}{c}\text { Scalability } \\
\text { (nodes) }\end{array}$ & \multicolumn{1}{|c|}{ Performance metrics } & \multicolumn{1}{|c|}{$\begin{array}{c}\text { MAC layer } \\
\text { standard } \\
\text { reasoning }\end{array}$} & $\begin{array}{c}\text { Modified } \\
\text { parameters and } \\
\text { modeling route } \\
\text { stabilities }\end{array}$ \\
\hline In [10] Samar R Das et al & $30 \& 60$ & Throughput, E2ED, NRL & Not Considered & not considered \\
\hline In [11]Bianchi et al & 50 & Other & Not Considered & not considered \\
\hline In [12] Daneshgram et al. & 20 & Throughput, E2ED, NRL & 802.11 & not considered \\
\hline $\begin{array}{l}\text { D. Malone } \text { et al. } \text { [2] \& } \\
\text { Engelstad } \text { et al. [3] }\end{array}$ & 20 & Other & $802.11 / 802.11 \mathrm{e}$ & not considered \\
\hline
\end{tabular}

Table 3: Our work

\begin{tabular}{|c|l|l|l|l|}
\hline \multirow{3}{*}{ Our work } & $\begin{array}{c}\text { Scalability } \\
\text { (Nodes) }\end{array}$ & $\begin{array}{c}\text { Performance } \\
\text { Metrics }\end{array}$ & $\begin{array}{c}\text { MAC Layer } \\
\text { Standard } \\
\text { Reasoning }\end{array}$ & \multicolumn{1}{|c|}{$\begin{array}{c}\text { Modified Parameters and Modeling } \\
\text { Route Stabilities }\end{array}$} \\
\cline { 2 - 5 } & 10 to 100 & $\begin{array}{l}\text { Throughput, } \\
\text { E2ED, NRL }\end{array}$ & $802.11 / 802.11 \mathrm{e}$ & $\begin{array}{l}\text { Modification in default parameters of } \\
\text { protocols and mathematical modeling is } \\
\text { done }\end{array}$ \\
\hline
\end{tabular}

\section{MODELING Route STABILITIES}

Modeling Route Stabilities of these three proactive routing protocols are determined depending upon their broadcast time interval $T_{B i}$, numerical computation have been carried out for each proactive routing protocols, shown in table 4,5,6. Route stabilities have been determined using equation (1). Suppose, we have 
two nodes $n a$ and $n b$. Node $n a$ transmits a packet at time $t_{j}$ which is received by $n b$ at time $t_{k}$. Link stability at node $n b$, for link $l$, at time $t_{k}$, for a particular broadcast interval $T_{B i}$ with respect to certain routing protocol parameters, Stability ${ }_{n b}^{l}\left(t_{k}\right)$ can be defined as follows:

$$
\text { Stability }_{n b}^{l}\left(t_{k}\right)=\frac{t_{k}(n a)-t_{j}(n a)}{T_{B i}}
$$

We believe that above equation is accompanied by the following constraints:

(i) $\forall \mathrm{k}>0$

(ii) $T_{B i} \neq 0$

(iii) $0<T_{B i}<1$ (Ideal Situation)

(iv) Stability ${ }_{n b}^{l}\left(t_{k}\right)<T_{h}$, where $\mathrm{T}_{\mathrm{n}}$ is a threshold value defined through specific parameter which varies from protocol to protocol.

In case of Trigger Updates between active links, $T_{B i}^{D S D V}$ varies according to state of the link. When link breaks, trigger messages are sent by the respective node by increasing NRL.

For $T_{B i}=0.01, T_{h} \leq 5$ and for $T_{B i}=0.07, \mathrm{~T}_{\mathrm{n}} \leq 0.71$, so $0.1 \leq T_{B i} \leq 0.7$

Table 4: Numerical computation for DSDV

\begin{tabular}{|l|l|l|}
\hline Parameters & Default value & Modified value \\
\hline$T_{B i}(s)$ & .0588 & 0.1176 \\
\hline$T_{h} \leq$ & 0.85 & 0.425 \\
\hline Stability & 0.017 & 0.0085 \\
\hline
\end{tabular}

Table 5: Numerical computation for OLSR

\begin{tabular}{|l|l|l|l|l|}
\hline Parameters & \multicolumn{2}{|c|}{ Inner scope } & \multicolumn{2}{c|}{ Outer scope } \\
\hline & default value & modified value & default value & modified value \\
\hline TTL & 2 & 2 & 255 & 255 \\
\hline $\mathrm{T}_{\mathrm{Bi}}(\mathrm{s})$ & $5 \mathrm{~s}$ & $1 \mathrm{~s}$ & $15 \mathrm{~s}$ & $3 \mathrm{~s}$ \\
\hline $\mathrm{T}_{\mathrm{h}} \leq$ & 0.01 & 0.05 & 0.0033 & 0.016 \\
\hline Stability & 0.00002 & 0.001 & 0.00006 & 0.003 \\
\hline
\end{tabular}

Table 6: Numerical computation for FSR

\begin{tabular}{|l|l|l|l|l|}
\hline Parameters & \multicolumn{2}{|c|}{ TC Messages } & \multicolumn{2}{c|}{ Hello Messages } \\
\hline & default value & modified value & default value & modified value \\
\hline $\mathrm{T}_{\mathrm{Bi}}(\mathrm{s})$ & $5 \mathrm{~s}$ & $3 \mathrm{~s}$ & $2 \mathrm{~s}$ & $1 \mathrm{~s}$ \\
\hline $\mathrm{T}_{\mathrm{h}} \leq$ & 0.01 & 0.016 & 0.025 & 0.05 \\
\hline Stability & 0.0002 & 0.0003 & 0.0005 & 0.001 \\
\hline
\end{tabular}

\section{SIMULATION RESULTS AND ANALYSIS}

In high scalabilities, (Figure 2 (a) and Figure 2(b)) MPRs technique achieves more optimization and efficiency, therefore both OLSR and M-OLSR overall produces high throughput among selected proactive protocols. After modification in all of three chosen protocols, OLSR produces the highest throughput both in DCF and EDCF because of frequent updating of routing messages results stabilized MPRs. DSDV achieves the second highest average throughput in DCF and EDCF as compared to the rest of two selected routing protocols. The reason for high efficiency of DSDV is due to incremental updates which are generated in case of any change in links of active routes. But in high scalabilities it fails to converge because of exchange of routing messages through flooding cause more overhead, and in high densities the rate of change is increased, thus causes more drop rates. On the other hand OLSRs throughput is more in high scalabilities of 80 nodes, 90 nodes and 100 nodes because MPRs provide more optimizations in high densities.

Whereas, FSR use only periodic updates for link status monitors and route updating. It is more suitable for hundreds and thousands of nodes, because fish-eye scopes with graded frequency mechanisms are best suited in very high densities. Frequent routing updating in FSR by reducing inner-scope and outer-scope intervals augments more throughputs in FSR-M as compared to FSR, but FSR-M fails to converge in EDCF comparative to that of FSR. Whereas, increasing the interval of triggered update generation in DSDV-M increase throughput in EDCF while throughput in DCF. 
In Figure.4 (c) and Figure.5 (d) E2ED of selected routing protocols is less in EDCF as compared to EDCF due to efficient stability checking of link in EDCF comparative to DCF. FSR attains the lowest routing latency in DCF as compared to DSDV and OLSR. Pure proactive approach route updating keeps overall routing latency low in FSR. Although in DSDV data remains for the time required for route settling to maintain correct information of routes before sending it to the destination, but this route settling time augments the delay. Therefore, DSDV produces the highest delay, moreover, it does not optimize network wise broadcasting (in DSDV only exchange of routing messages are only performed through flooding, as compared to MPRs of OLSR which reduce number of retransmissions and scope updates of FSR by using graded frequency mechanism).

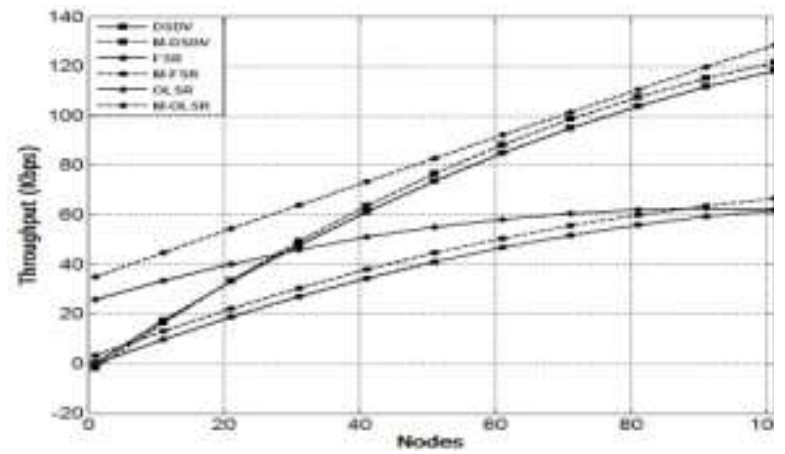

Figure 2 (a): Throughput 802.11 DCF

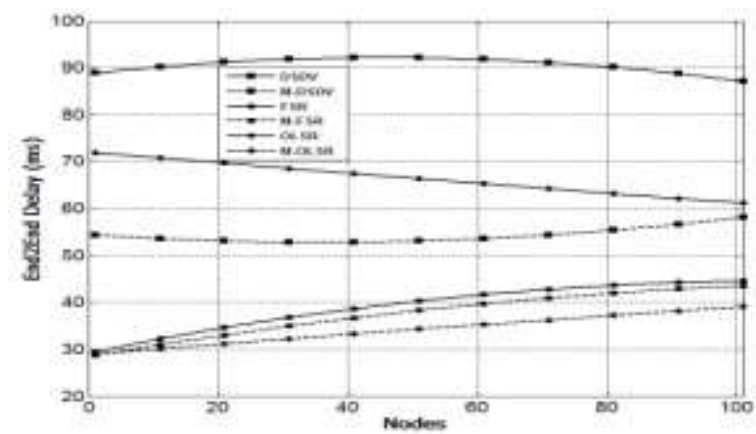

Figure 3 (a): End to end delay 802.11 DCF

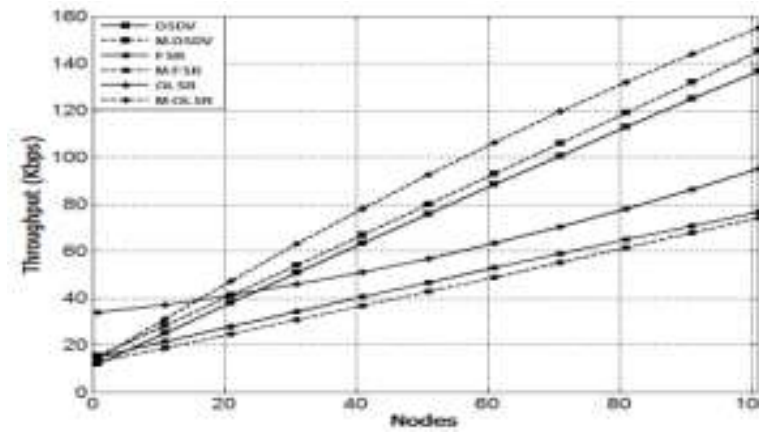

Figure 2 (b): Throughput 802.11 EDCF

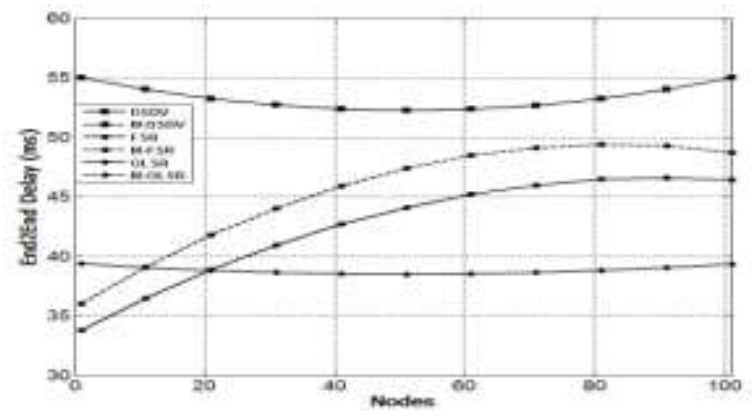

Figure 3 (b): End to end delay 802.11 EDCF

DSDV-M achieves less routing latency as ompared to DSDV in case of DCF. While DSDV-M and DSDV possess equal routing delay in EDCF, because efficient mechanism of 802.11e EDCF helps to reduce E2ED. Same is the case of OLSR, where OLSR-M achieves lowest delay as compared to OLSR in EDCF, while this delay is more in EDCF. As FSR-Ms periodic intervals for scope routing is reduced, therefore it attains low E2ED in both DCF and EDCF, moreover it does not update routing information instantly after detection of any change in the network unlike DSDV and OLSR, therefore EDCF mechanism not effects too much to improve its performance.
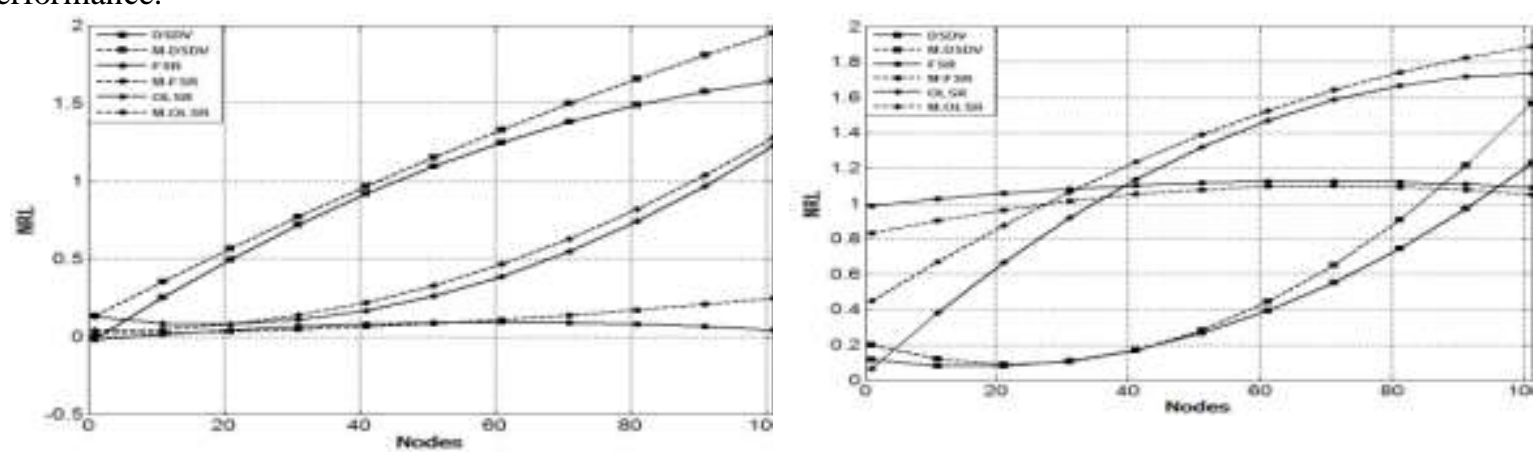

Figure 4: High NRL after modifications

All selected routing protocols have attained high NRL referring to Figure 6 after modifications. The rate for successive routing messages exchange is highest in OLSR as compared to rest of protocols (clearly mention in table) and, therefore, it generates high routing load. As FSR does not trigger any routing messages in case of link breakage and relies only on periodic updating, thus it produces lowest NRL in DCF. Furthermore, DSDV produces lowest routing messages in case of EDCF as compared to OLSR and FSR as shown in Fig.6, because of efficient information of link connectivity reduces incremental updates. TTL value in ring search algorithm 
increases while increase in network density is observed, this leads to increase in broadcast routing packets during route discovery as increasing NRL behavior in FSR.

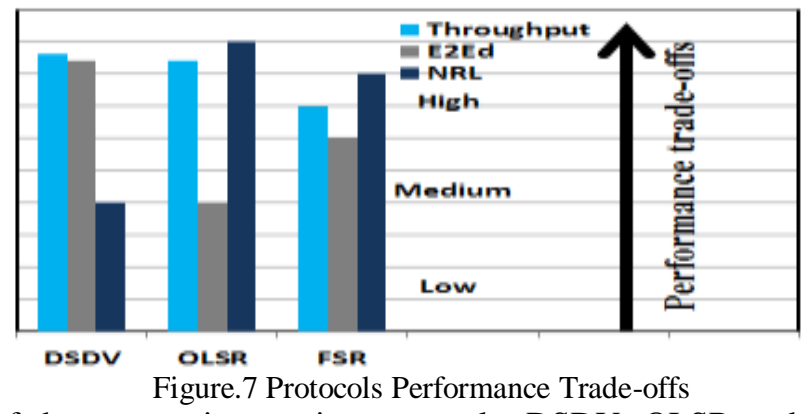

Performance Trade-offs of three proactive routing protocols; DSDV, OLSR and FSR as shown in Figure 7, according to three performance metrics are carried out based on simulation results analysis. DSDV achieves enhanced and high throughput with trade-off between E2ED but reduce routing overhead is observed by using incremental. OLSR outperforms as concerned to its lowest E2ED observed with the increase in throughput and normalized routing load. FSR produces more normalized routing load with a minimum trade-off between other two performance metrics i.e. throughput and E2ED.

Table 7: Performance comparison

\begin{tabular}{|l|c|c|}
\hline Protocols & Default parameters & Modified parameters \\
\hline DSDV & Tbi=15 $\mathrm{s}$ or $8 / 15=.0588 \mathrm{~s}$ & Tbi $b i 0 \mathrm{~s}$ or .1176 s \\
\hline OLSR & TC_Messages $=5 \mathrm{~s}$ & TC_Messages $=3 \mathrm{~s}$ \\
& Hello_Messgaes $=2 \mathrm{~s}$ & Hello_Messgaes $=1 \mathrm{~s}$ \\
\hline FSR & Tbi $=5 \mathrm{~s}$ & T $b i=1 \mathrm{~s}$ \\
\hline
\end{tabular}

\section{CONCLUSION AND FUTURE WORK}

In this paper, we have estimated and matched the performance of three extensively utilized proactive protocols; DSDV, OLSR and FSR. Total normalized routing load achieved by a protocol is centered upon two changing aspects; control traffic produced by control packets and data traffic accelerated over and done with routes of non-optimal path lengths. Consequently, for evaluating the route stabilities of these protocols in dense networks and with different broadcast intervals of each routing protocol, we have varied different scalability scenarios. In lieu of analysis, three performance parameters; E2ED, NRL and throughput are worked out by using NS-2. In conclusion, we perceived that OLSR is additionally scalable for the reason of bargain in routing overhead due to MPRs and lowest E2ED, as OLSR permits retransmission via MPRs. On the other influence, FSR is supplementary appropriate for extraordinary network loads owed to scope routing over GF (no flooding), that decreases broadcasting storm, as a result it saves additional bandwidth and accomplishes high throughput when data traffic upturns in high scalabilities.

In future, we are concerned to evaluate the reactive routing protocols under same scalability scenarios but with varying mobilities and under route stabilities computed through proposed equation(1).

\section{REFERENCES}

[1] Peter Clifford et al.,"Modeling 802.11e for data traffic parameter design" Hamilton Institute, Ireland.

[2] D. Malone, et al., "Modeling the 802.11 distributed coordination function in non-saturated heterogeneous conditions', IEEE/ACM Transactions on Networking. 2007.

[3] P.E. Engelstad, et al., 'Non-saturation and saturation analysis of IEEE 802.11e EDCA with starvation prediction', ACM International Symposium, 2005.

[4] J. Hu, et al., 'A comprehensive analytical model for IEEE 802.11e QoS differentiation schemes under unsaturated traffic loads, in: Proceedings of the ICC08', 2008.

[5] C. E. Perkins and P. Bhagwat, "Highly dynamic Destination-Sequenced Distance-Vector routing (DSDV) for mobile computers," SIGCOMM Comput. Commun. Rev.,vol. 24, pp. 234-244, 1994.

[6] G. Pei, et al., "Fisheye State Routing in Mobile Ad Hoc Networks," in ICDCS Work-shop on Wireless Networks and Mobile Computing, 2000, pp. D71-D78.

[7] M. Gerla, et al., IETF Draft-01, "Fisheye State Routing Protocol (FSR) for Ad Hoc Networks"," 2000

[8] T. Clausen and P. Jacquet, "Optimized Link State Routing Protocol (OLSR)", 2003.

[9] T. Clausen and P. Jacquet, IETF RFC-3626, "Optimized Link State Routing Protocol OLSR," The Internet Society http://www. ietf. org/rfc/rfc3626. txt, 2003.

[10] Samir R. Das, et al.,"Simulation-based performance evaluation of routing protocols for mobile ad hoc networks".

[11] G. Bianchi, Performance analysis of the IEEE 802.11 distributed coordination function, IEEE Journal on Selected Areas in Communications 18 (3) (2000).

[12] F. Daneshgaran, et al., 'Unsaturated throughput analysis of IEEE 802.11 in presence of non-ideal transmission channel and capture effects', IEEE Transactions on Wireless Communications 7 (4) (2008) 12761286. 